\title{
Investigation of PDMS-gold nanoparticle composite films for plasmonic sensors
}

\author{
Attila Bonyár ${ }^{1}, Z^{2}$ anett Izsold ${ }^{1}$ \\ ${ }^{1}$ Department of Electronics Technology \\ Budapest University of Technology and Economics \\ Budapest, Hungary \\ bonyar@ett.bme.hu \\ László Himics ${ }^{2}$, Miklós Veres ${ }^{2}$ \\ ${ }^{2}$ Institute for Solid State Physics and Optics, Wigner Research Centre for Physics of the Hungarian Academy of Sciences, \\ Budapest, Hungary \\ István Csarnovics ${ }^{3}$ \\ ${ }^{3}$ Department of Experimental Physics \\ University of Debrecen \\ Debrecen, Hungary
}

\begin{abstract}
Poly(dimethylsiloxane) (PDMS)-gold nanoparticle composite films were synthetized in situ by using a simple method based on the reduction of chloroauric acid $\left(\mathrm{HAuCl}_{4}\right)$ by the PDMS membrane. The technological parameters which affect the gold nanoparticle formation on the membrane (namely the concentration of the $\mathrm{HAuCl}_{4}$ solution, the ratio of curing agent, the incubation time and the temperature) were investigated, the resulting nanoparticle films were characterized with optical spectrophotometry. The possibility to utilize the nanocomposite membranes as sensing elements in plasmonics sensors (based on localised surface plasmon resonance - LSPR) and as surface enhanced Raman spectroscopy (SERS) substrates is discussed in detail
\end{abstract}

Keywords— PDMS, LSPR, SERES, gold nanoparticles, plasmonics, AFM.

\section{INTRODUCTION}

Gold nanoparticles are widely used as signal amplification elements in various electrochemical and optical sensor applications [1]. This includes for example the conjugation of Au NPs with biomolecules as biological tags in biosensoric application, but Au NP modified sensor transducer surfaces (e.g. thin films) are also quite frequent. The nanoparticles on the transducer surface can in one hand increase the effective electrode area in electrochemical applications, while in optical sensors the so called localized surface plasmon resonance (LSPR) phenomenon - the collective oscillations of delocalized electrons in response to an external electric field - can be utilized for a large variety of sensing purposes (chemical sensors, gas sensors, biosensors, etc.) by measuring changes in the refractive index of the surrounding media with high sensitivity [2,3]. Localized surface plasmons can also be used to increase the effectivity of Raman scattering: surface enhanced Raman spectroscopy (SERS) is an analytical method which can significantly increase the yield of Raman scattering of target molecules adsorbed on the surface of metallic nanoparticles [4]. Depending on the nanoparticles and some other conditions the enhancement factor can reach the order of $10^{10}-10^{11}$, which would enable even the detection of single molecules on the surface.

Au NPs can be synthesized in several ways [5]. Hereby we aim to investigate a simple method, which is based on the reduction of chloroauric acid $\left(\mathrm{HAuCl}_{4}\right)$ on a PDMS membrane [6]. Poly(dimethylsiloxane) (PDMS) is one of the most widely used polymer materials for fabricating microfluidic chips due to its transparency, outstanding elasticity, good thermal and oxidative stability and ease of fabrication [6]. During the nanoparticle synthesis process, the residual curing agent in the PDMS matrix itself is used to reduce $\mathrm{HAuCl}_{4}$ to gold nanoparticles. The advantage of this method compared or other fabrication technologies, is that the synthetized nanoparticles are readily available on the PDMS membrane - no further binding is required - and this way they could be directly applied as integrated transducers in a polymer microfluidic cell.

The aim of the current work is the investigation and optimization of the technological parameters of such PDMS-gold nanocomposite membranes in order to fabricate cheap but sensitive photonic elements for both LSPR and SERS applications. 


\section{MATERIALS AND METHODS}

\section{A. PDMS Preparation}

Poly(dimethylsiloxane) (PDMS) blocks, with a thickness of $5 \mathrm{~mm}$ were prepared by mixing SYLGARD ${ }^{2} 184$ silicone elastomer with its corresponding curing agent in 1:10 and 1:5 mass ratio $(\eta)$, respectively. The mixture was degassed in a vacuum exsiccator then poured to a mold to form a $10 \mathrm{~cm}$ x $10 \mathrm{~cm}$ x $5 \mathrm{~mm}$ film. To increase the speed of polymerization the molding form was placed to a ceramic oven for $30 \mathrm{~min}$ at $120^{\circ} \mathrm{C}$. Before the subsequent nanocomposite preparation, the PDMS film was cut into smaller blocks (as can be seen in Fig. 1).

\section{B. PDMS-Au nanocomposite preparation}

To prepare the PDMS-gold nanoparticle composite films $100 \mu \mathrm{l}$ of $0.5 \%$ (m/V) chloroauric acid ( $\mathrm{HAuCl}_{4}$, from Sigma Aldrich) was pipetted on top of the PDMS blocks. The incubation time and temperature was varied, as collected in Table 1 . The samples under incubation were put into a hermetically sealed Petri dish, along with drops of water, to avoid the evaporation of the $\mathrm{HAuCl}_{4} \mathrm{Solution}_{\text {. }}$ After the given incubation time the samples were rinsed with deionized water, dried then were subjected to optical spectroscopy immediately.

Table 1. The technological parameters of the investigated nanocomposite samples. Curing agent/monomer ratio of PDMS ( $\eta$ ), incubation time and temperature.

\begin{tabular}{|c|c|c|c|}
\hline $\begin{array}{c}\text { Sample } \\
\text { ID }\end{array}$ & $\eta$ & Time & Temp \\
\hline$\# 1$ & $1: 10$ & 4 days & $22^{\circ} \mathrm{C}$ \\
\hline$\# 2$ & $1: 10$ & 7 days & $22^{\circ} \mathrm{C}$ \\
\hline$\# 3$ & $1: 10$ & 7 days & $22^{\circ} \mathrm{C}$ \\
\hline$\# 4$ & $1: 5$ & $23 \mathrm{~h}$ & $22^{\circ} \mathrm{C}$ \\
\hline$\# 5$ & $1: 5$ & 5 days & $22^{\circ} \mathrm{C}$ \\
\hline$\# 6$ & $1: 5$ & 5 days & $22^{\circ} \mathrm{C}$ \\
\hline$\# 7$ & $1: 5$ & 4 days & $22^{\circ} \mathrm{C}$ \\
\hline$\# 8$ & $1: 5$ & 4 days & $22^{\circ} \mathrm{C}$ \\
\hline$\# 9$ & $1: 10$ & $4 \mathrm{~h}$ & $65^{\circ} \mathrm{C}$ \\
\hline$\# 10$ & $1: 5$ & $4 \mathrm{~h}$ & $65^{\circ} \mathrm{C}$ \\
\hline$\# 11$ & $1: 10$ & $5 \mathrm{~h}$ & $65^{\circ} \mathrm{C}$ \\
\hline$\# 12$ & $1: 5$ & $5 \mathrm{~h}$ & $65^{\circ} \mathrm{C}$ \\
\hline$\# 13$ & $1: 10$ & $2 \mathrm{~h}$ & $80^{\circ} \mathrm{C}$ \\
\hline$\# 14$ & $1: 5$ & $2 \mathrm{~h}$ & $80^{\circ} \mathrm{C}$ \\
\hline \# 15 & $1: 10$ & $3 \mathrm{~h}$ & $80^{\circ} \mathrm{C}$ \\
\hline \# & $1: 5$ & $3 \mathrm{~h}$ & $80^{\circ} \mathrm{C}$ \\
\hline
\end{tabular}

\section{Spectrophotometry}

To test the plasmonics response of the specimens optical spectroscopy measurements were performed with an Avantes Avaspec 2048-4DT spectrometer and an Avantes Avalight DHS halogen light source between $400 \mathrm{~nm}$ and $750 \mathrm{~nm}$.

\section{SERS}

SERS measurements were performed with a Renishaw 1000 micro-Raman spectrometer. The $488 \mathrm{~nm}$ line of a DPSS (diode pumped solid state) laser was used for the excitation and the excitation spot was focused into a spot having diameter of 1 micron. The spectra were recorded with $10 \mathrm{~s}$ integration time. The SERS enhancement was tested using benzophenon-isopropyl alcohol solution by dripping the same volume onto the substrate so that the same drop covered partially both the nanoparticle coated region and the clean Si wafer substrate. The latter was used for the reference measurements. 


\section{RESULTS AND DISCUSSIONS}

\section{A. The effect of the investigated preparation parameters}

The prepared samples are presented in Fig. 1. By comparing their color and their measured absorbance spectra we can make the following general observations regarding the effect of the preparation parameters on the density of the prepared Au NP-PDMS films. Increasing the curing agent/monomer ratio yielded denser and larger nanopartilces (for the same incubation times/temperatures). This is illustrated in Fig. 2 for samples 11 and 12, which both were prepared at $65{ }^{\circ} \mathrm{C}$ for 5 hours incubation. Denser nanoparticle layers, and also larger average nanoparticle sizes shift the absorbance peak to higher wavelengths. (AFM investigations confirmed, that both the particle size and density increase by increasing the curing agent/monomer ratio - data not shown.)

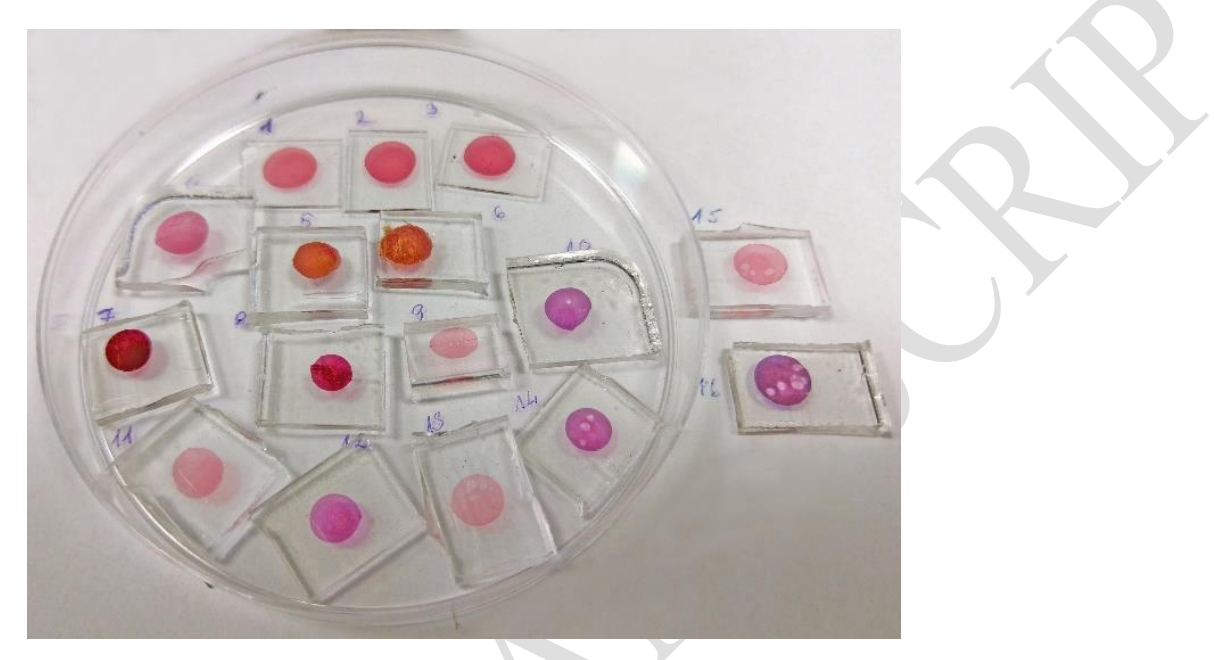

Fig. 1. The tested samples, for the preparation parameters see Table 1.

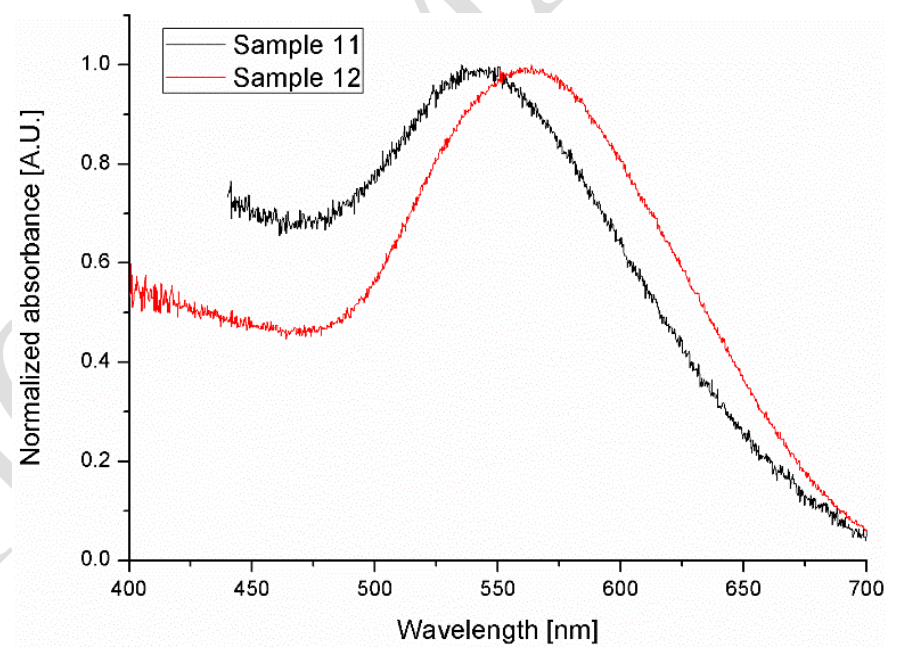

Fig. 2. Normalized absorbance spectra of the samples 11 and 12 measured in air. Both samples were incubated for $5 \mathrm{~h}$ at $65{ }^{\circ} \mathrm{C}$, the only difference is in their curing agent/monomer ratio (1:10 and 1:5 respectively).

The main reason for testing higher incubation temperatures is that the formation of nanoparticles at ambient temperature is very slow. Fig. 3 illustrates the effect of process parameters for the $\eta=1: 5$ samples. At higher temperatures $\left(65-80{ }^{\circ} \mathrm{C}\right)$ even after 2 hours we can obtain denser particle layer than after 4 days of incubation at room temperature. $80{ }^{\circ} \mathrm{C}$ was found to be too high for incubation, as can be seen in Fig. 1 for samples 13-16, after 2-3 hours small air bubbles appeared in the incubation solution attached to the PDMS surface due to evaporation, which resulted in uneven coverage. Thus no longer incubation times were investigated at $80{ }^{\circ} \mathrm{C}$. At $65^{\circ} \mathrm{C}$ bubble formation was not observable, the measured absorbance peak showed a steady shift to longer wavelength with the incubation time. 


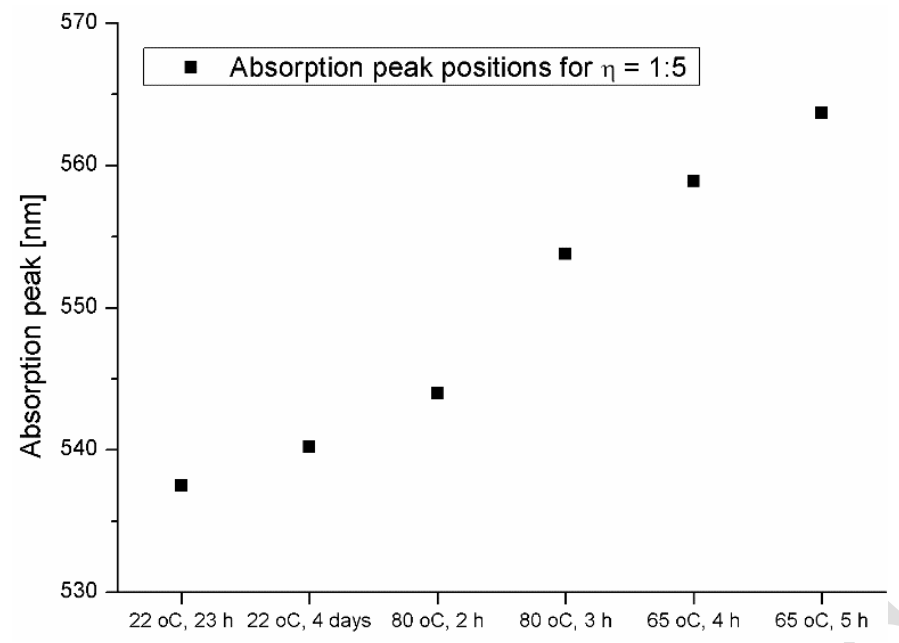

Fig. 3. Position of the absorbance peak of the samples with 1:5 curing agennt/monomer ratio, measured in air. The peak positions are arranged in increasing order for the sake of comparison.

Conclusively, higher temperatures could be successfully utilized to significantly shorten the incubation time of dense nanoparticle layer formation.

\section{B. LSPR measurements}

In order the test the potential application of the nanocomposite films as LSPR sensor elements the bulk refractive index sensitivities of the samples were obtained by measuring their absorbance spectra in air $(n=1)$ and water $(n=1.33)$, respectively. Fig. 4 illustrates the results for sample 5. The bulk refractive index sensitivity is defined as the shift of the absorbance peak caused by the changes in the refractive index of the surrounding media. The samples performed under expectations, the absolute peak shifts varied between 0-8.7 $\mathrm{nm}$, corresponding to maximum refractive index sensitivities of around $26 \mathrm{~nm} / \mathrm{RIU}$. The small obtained sensitivities (and the fact that most of the samples yielded nearly no response at all) confirm, that instead of surface-bound nanoparticles, the fabricated films really are nanocomposites, with Au NPs fully, or partially embedded into the upper layer of the PDMS block. From the point of localized surface plasmon resonance sensing, partial nanoparticle embedment in the PDMS can decrease the sensitivity significantly.

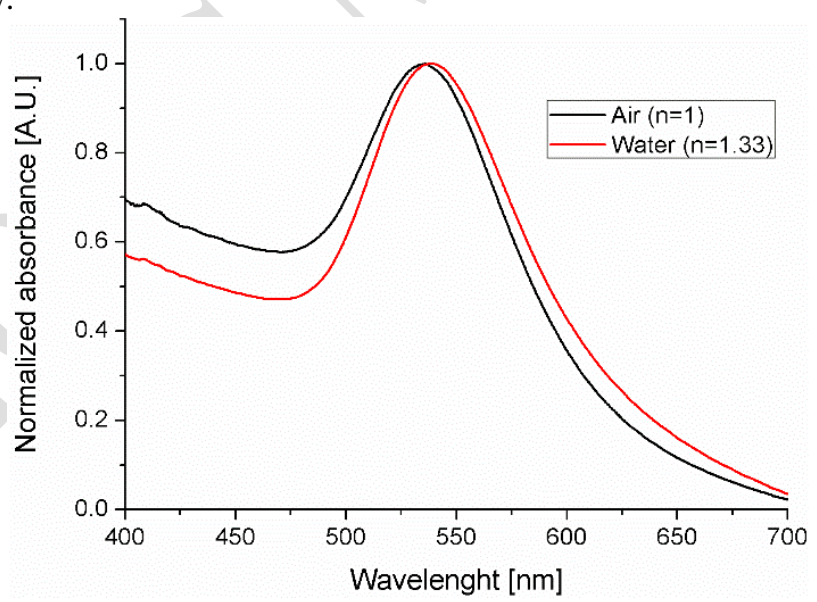

Fig. 4. Normalized absorbance specta of sample 5, measured in air and water, respectively.

\section{SERS measurements}

To test the potential application of the samples as SERS substrates, a benzophenon-isopropyl alcohol solution was used to test the response of the nanocomposite films in contrast to polished Si substrate, which was used as reference. Up to this point, only sample 12 and 16 were tested, as presented in Fig.5. 


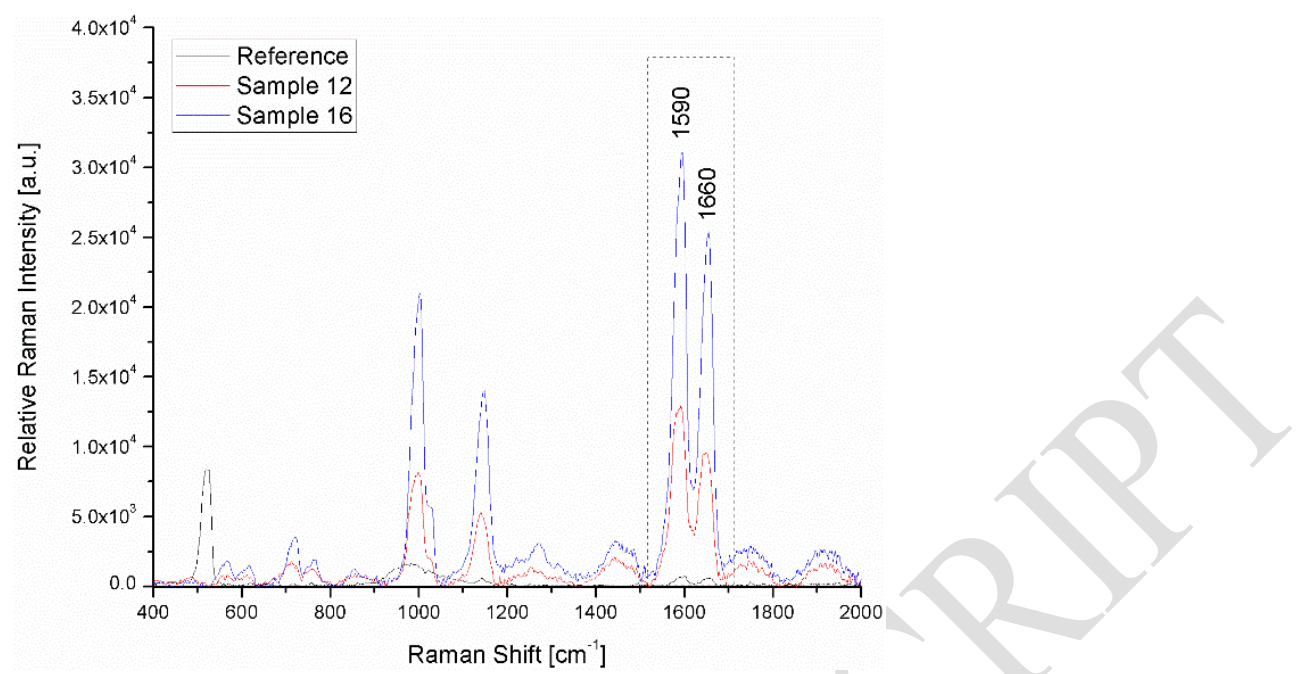

Fig. 5. Comparison of the Raman (measured with the reference substrate) and SERS (measured with samples 12 and 16) spectra of the benzophenon-isopropyl alcohol test solution. The bracket shows the two charecteristic peaks whoch were used for the evaluation of the SERS enhancement.

As can clearly be seen, both samples increase the Raman yield of the test solution significantly. To calculate SERS enhancement, the peaks found at $1590 \mathrm{~cm}-1$ and $1660 \mathrm{~cm}-1$ were used. (Here we use SERS enhancement as the relative increase in the Raman intensity caused by the nanostructured surfaces, sompared to the reference). The results are collected in Fig. 6. For comparison, the measurements were repeated on a commercially available SERS substrate, Klarite 302 from Mesophotonics, which was also added to Fig. 6. Both of the nanocomposite samples performed better than the reference SERS substrate. Sample 16, with its average enhancement of around 9-10x outperformed the Klarite 302 substrate around 5 times. Further investigation of the nanocomposite films as SERS substrates will be performed since it seems they prove to be rather promising candidates for SERS applications.

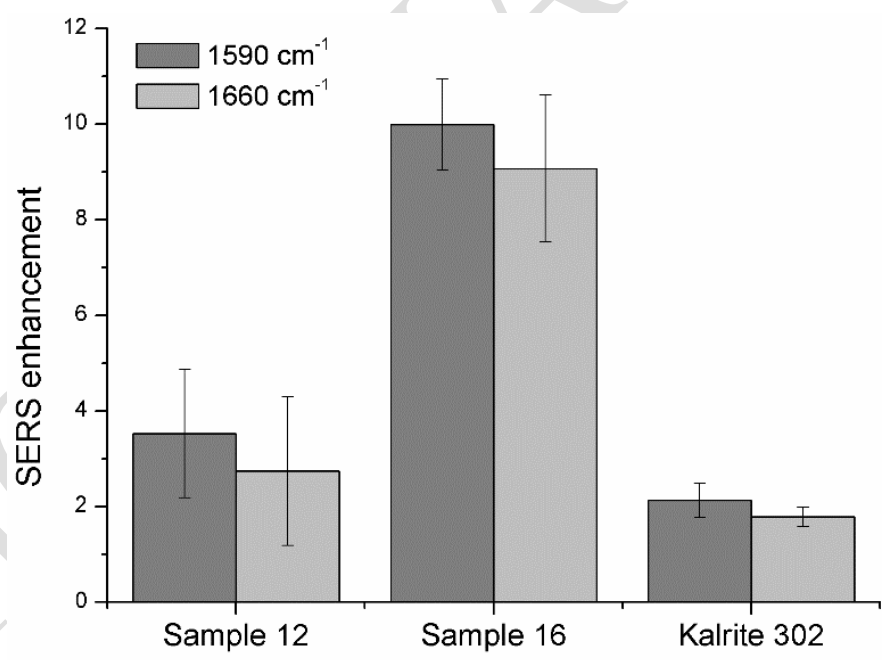

Fig. 6. SERS enhancements measured on samples 12 and 16, compared to a commercially available SERS substrate, Kalrite 302.

\section{CONCLUSIONS}

PDMS-gold nanoparticle composite films were prepared by the direct reduction of chloroauric acid by the residual curing agent of the polymer. We showed that by increasing the temperature during incubation the process time can be significantly decreased and dense particle layers can be formed in the matter of $2-5$ hours (at $65{ }^{\circ} \mathrm{C}$ ). The test of the nanocomposites as LSPR sensor elements showed that the particles are at least partially embedded in the PDMS, since the measured refractive index sensitivities were very low $(<26 \mathrm{~nm} / \mathrm{RIU})$. However, the possible application of the nanocomposite films as SERS substrates seems to be promising and will be subjected to further investigations. 


\section{ACKNOWLEDGMENT}

This work was supported by the TÁMOP 4.2.4.A/2-11-1-2012-0001 (Nemzeti Kiválóság Program) and the TAMOP 4.2.2.A11/1/KONV-2012-0036 Project, which are cofinanced by the European Union and European Social Fund. The authors acknowledge the financial support by the grant GINOP- 2.3.2-15-2016-00041. Attila Bonyár and Miklós Veres is grateful for the support of the János Bolyai Research Scholarship of the Hungarian Academy of Sciences.

\section{REFERENCES}

[1] Xiaodong Cao, Yongkang Ye, Songqin Liu “Gold nanoparticle-based signal amplification for biosensing”, Analytical Biochemistry, Vol. 417, pp. 1-16, 2011.

[2] J. Zhao, X. Zhang, CR. Yonzon, AJ. Haes, RP. Van Duyne, Localised surface plasmon resonance biosensors, Nanomedicine, 2 (2006) $219-228$.

[3] A. Dimitrev, Nanoplasmonic Sensors, Springer, 2012

[4] S. Link, M. A. El-Sayed, Shape and sized dependence of radiative, non-radiative and photothermal properties of gold nanocrystals, Int. Reviews in Physical Chemistry, 19 (2000) 409-453.

[5] Pengxiang Zhao, Na Li, Didier Astruc "State of the art in gold nanoparticle synthesis", Coordination Chemistry Reviews, Vol. 257 pp. 638- 665, 2013

[6] Qing Zhang, Jing-Juan Xu, Yan Liu and Hong-Yuan Chen, " In-situ synthesis of poly(dimethylsiloxane)-gold nanoparticles composite films and its application in microfluidic systems ", Lab Chip, Vol. 8, pp. 352-357, 2008, 